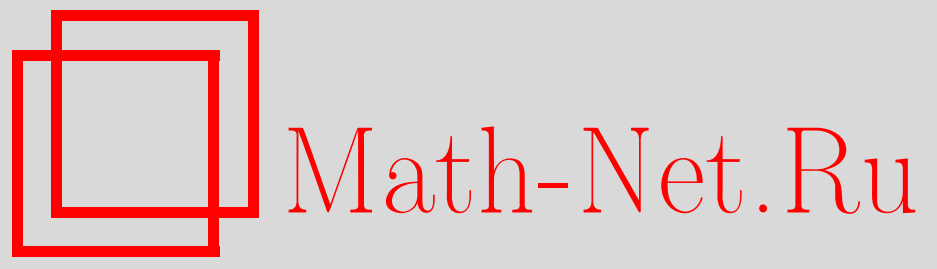

А. А. Гущин, О расширении понятия $f$-дивергенции, Теория вероятн. и ее примен., 2007, том 52, выпуск $3,468-489$

DOI: https://doi.org/10.4213/tvp74

Использование Общероссийского математического портала MathNet.Ru подразумевает, что вы прочитали и согласны с пользовательским соглашением

http://www . mathnet.ru/rus/agreement

Параметры загрузки:

IP : 35.173 .137 .237

26 апреля 2023 г., 14:02:18

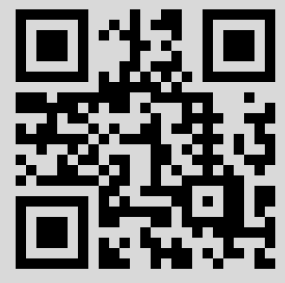




\section{О РАСШИРЕНИИ ПОНЯТИЯ $f$-ДИВЕРГЕНЦИИ ${ }^{1)}$}

Для полунепрерывной снизу выпуклой функции $f: \mathbf{R} \rightarrow \mathbf{R} \cup$ $\{+\infty\}, \operatorname{dom} f \subseteq \mathbf{R}_{+}$, дается определение и изучаются свойства $f$-дивергенции конечно-аддитивных функций множества $\mu$ и $\nu$, заданных на измеримом пространстве $(\Omega, \mathscr{F})$. В случае, когда $f$ конечна на $(0,+\infty)$, а $\mu$ и $\nu$ - вероятностные меры, наше определение эквивалентно классическому определению $f$-дивергенции, введенному И. Чисаром. В качестве применения получен результат о достижении минимума $f$-дивергенции на множестве $\mathscr{Z}$ пар вероятностных мep.

Ключевые слова и фразы: $f$-дивергенция, конечно-аддитивная функция множества.

1. Введение. Понятие $f$-дивергенции двух вероятностных мер (где $f:(0,+\infty) \rightarrow \mathbf{R}-$ произвольная выпуклая функция) было введено И. Чисаром в 1963 г. в работе [5]. Напомним определение $f$-дивергенции. В силу выпуклости $f$ предел $\lim _{y \downarrow 0} f(y)$ существует и может принимать конечное значение либо $+\infty$; доопределим $f$ в нуле по непрерывности. Аналогично, существует предел

$$
\frac{f(\infty)}{\infty}:=\lim _{y \uparrow+\infty} \frac{f(y)}{y} \in \mathbf{R} \cup\{+\infty\} .
$$

Положим

$$
F(s, t)=\left\{\begin{array}{lllll}
t f\left(\frac{s}{t}\right), & \text { если } s \geqslant 0 \quad \text { и } t>0, \\
s \frac{f(\infty)}{\infty}, & \text { если } s>0 \text { и } t=0, \\
0, & \text { если } s=0 \text { и } t=0 .
\end{array}\right.
$$

Пусть на измеримом пространстве $(\Omega, \mathscr{F})$ заданы вероятностные меры $\mathrm{P}$ и $\mathrm{Q}$, доминируемые $\sigma$-конечной мерой $\varrho$. Тогда $f$-дивергенцией мер $\mathrm{P}$ и $\mathrm{Q}$ называется величина

$$
\mathscr{J}_{f}(\mathrm{P}, \mathrm{Q})=\int_{\Omega} F\left(\frac{d \mathrm{P}}{d \varrho}, \frac{d \mathrm{Q}}{d \varrho}\right) d \varrho
$$

* Математический институт им. В. А. Стеклова РАН, ул. Губкина, 8, 119991 Москва, ГСП-1, Россия; e-mail: gushchin@mi.ras.ru

1) Работа выполнена при поддержке РФФИ (грант №05-01-00944). 
Интеграл в (2) корректно определен, его значение не зависит от выбора доминирующей меры $\varrho$ и лежит в $\mathbf{R} \cup\{+\infty\}$.

Частными случаями $f$-дивергенции являются расстояние по вариации $\|\mathrm{P}-\mathrm{Q}\|(f(y)=|y-1|)$, квадрат расстояния Хеллингера $\rho^{2}(\mathrm{P}, \mathrm{Q})\left(f(y)=\frac{1}{2}(\sqrt{y}-1)^{2}\right)$, относительная энтропия, или информация Кульбака-Лейблера, $D(\mathrm{P} \| \mathrm{Q})(f(y)=y \ln y)$, относительная энтропия $D(\mathrm{Q} \| \mathrm{P})(f(y)=-\ln y)$.

Аналогично определяется $f$-дивергенция $\mathscr{J}_{f}(\mu, \nu)$ конечных (неотрицательных) мер $\mu$ и $\nu$. Если хотя бы одна из мер $\mu$ и $\nu \sigma$-конечна, соответствующий интеграл может быть не определен (одновременно для всех $\varrho)$ или быть равен $-\infty$.

Изложение теории $f$-дивергенций и ее применения в задачах математической статистики можно найти в монографии Ф. Лизе и И. Вайды [23]. В последнее время стали появляться работы, где теория $f$-дивергенций используется для решения некоторых задач финансовой математики, например, задачи нахождения верхних и нижних цен некоторых опционов европейского типа [18].

Другой областью применения теории $f$-дивергенций в финансовой математике является задача максимизации ожидаемой полезности (см. [13], [14], [12]). Применениями именно к этой задаче вызвана данная работа. Во-первых, мы расширяем область определения $f$-дивергенции со счетно-аддитивных мер до ограниченных конечно-аддитивных функций множества (для краткости называемых нами конечно-аддитивными мерами), принимающих неотрицательные значения. Соответствующие результаты были анонсированы автором в [17]. Во-вторых, мы рассматриваем более широкий класс функций $f$, а именно, считаем, что $f$ - полунепрерывная снизу выпуклая функция на $\mathbf{R}_{+}$со значениями в $\mathbf{R} \cup\{+\infty\}$, т.е. мы отказываемся от конечности $f$ в точках интервала $(0,+\infty)$. В задаче максимизации ожидаемой полезности это отвечает отказу от условий Инада на функцию полезности, см. п. 4. Использование результатов данной статьи в задаче максимизации полезности автор намерен подробно обсудить в отдельной публикации.

Статья организована следующим образом. В п. 2 дается определение $f$-дивергенции и формулируется основной результат работы - теорема 1, в которой приведены основные свойства $f$-дивергенции. В п. 3 рассматривается задача о достижении минимума $f$-дивергенции $\mathscr{J}_{f}(\mathrm{P}, \mathrm{Q})$ на множестве $(\mathrm{P}, \mathrm{Q}) \in \mathscr{Z}$ пар вероятностных мер. Основные результаты этого пункта - теорема 2 и следствие 2 , в доказательстве которых ключевую роль играет расширение области определения $f$-дивергенции до конечно-аддитивных мер. Следствие 2 обобщает недавний результат Х. Фёльмера и А. Гундель [12], доказанный другим методом. Пункт 4 содержит вспомогательные результаты. Теорема 1 доказана в п. 5. В заключительном п. 6 обсуждается обобшение пред- 
ложенного подхода к определению $f$-дивергенции на случай конечного числа конечно-аддитивных мер, а именно, для множества $D \subseteq \mathbf{R}^{k}$ вводится понятие $D$-дивергенции $\mathscr{J}_{D}\left(\mu_{1}, \ldots, \mu_{k}\right) ; f$-дивергенция есть частный случай $D$-дивергенции, отвечающий $k=2$ и специальному выбору множества $D=D(f)$.

В выпуклом анализе принято доопределять функции, заданные на подмножестве линейного пространства, до функций, заданных на всем пространстве, полагая их равными $+\infty$ вне исходной области определения. Мы также считаем, что функция $f$, для которой будет определяться $f$-дивергенция, задана на $\mathbf{R}$, причем всегда $f(y)=+\infty$ при $y<0$. Соответственно, $f$-дивергенция $\mathscr{J}_{f}(\mu, \nu)$ будет определена для произвольных конечно-аддитивных мер $\mu, \nu$, но при этом $\mathscr{J}_{f}(\mu, \nu)=+\infty$, если хотя бы одна из мер принимает не только неотрицательные значения. Такой выбор основного объекта исследования объясняется приложениями, которые мы имеем в виду. В других приложениях может возникнуть необходимость рассмотрения дивергенций знакопеременных мер, см., например, работу [7], где рассматривалась $\gamma$-дивергенция знакопеременной счетно-аддитивной меры и вероятностной меры; $\gamma$ имеет тот же смысл, что и $f$, но уже может принимать конечные значения на отрицательной полуоси. Следует подчеркнуть, однако, что рассматриваемое нами в п. 6 понятие $D$-дивергенции даже в случае $k=2$ является значительно более общим по сравнению с $\gamma$-дивергенцией и устраняет всякое различие между положительными и отрицательными значениями мер; если же рассматривать $f$ - и $D$-дивергенции мер с неотрицательными значениями, то эти два понятия, по существу, совпадают (для $k=2$ ).

Для удобства читателя напомним терминологию и элементарные факты из выпуклого анализа, постоянно используемые в работе, см., например, [1, § 2.6] или [11, гл. 1].

Пусть $X$ и $X^{\prime}$ - два линейных пространства, двойственных относительно билинейной формы $\langle\cdot, \cdot\rangle$ (в частности, в качестве одного из пространств можно взять банахово пространство, а в качестве другого его сопряженное). Здесь пространства $X$ и $X^{\prime}$ считаются наделенными топологиями $\sigma\left(X, X^{\prime}\right)$ и $\sigma\left(X^{\prime}, X\right)$ соответственно, превращающими их в хаусдорфовы локально выпуклые топологические векторные пространства. Пусть задана функция $f: X \rightarrow \mathbf{R} \cup\{-\infty\} \cup\{+\infty\}$. Эффективным множеством и надграфиком $f$ называются множества

$$
\operatorname{dom} f:=\{x \in X: f(x)<+\infty\}
$$

и

$$
\text { epi } f:=\{(x, \alpha) \in X \times \mathbf{R}: \alpha \geqslant f(x)\}
$$

соответственно. Функция $f$ выпукла тогда и только тогда, когда ее надграфик является выпуклым множеством. Функция $f$ полунепрерывна 
снизу на $X$ тогда и только тогда, когда ее надграфик есть замкнутое множество (относительно произведения топологий на $X$ и $\mathbf{R}$ ). Функция $f$ называется собственной, если она не принимает значение $-\infty$ и $\operatorname{dom} f \neq \varnothing$. Субдифференциал $f$ в точке $x \in X$ обозначается $\partial f(x)$; напомним, что

$$
\partial f(x)=\left\{x^{\prime} \in X^{\prime}: f(y) \geqslant f(x)+\left\langle y-x, x^{\prime}\right\rangle \text { для любого } y \in X\right\} .
$$

Сопряженной к $f$ или преобразованием Фенхеля называется функция $f^{*}$ на пространстве $X^{\prime}$, задаваемая равенством

$$
f^{*}\left(x^{\prime}\right)=\sup _{x \in X}\left[\left\langle x, x^{\prime}\right\rangle-f(x)\right] .
$$

Сопряженная функция полунепрерывна снизу и выпукла. Для собственной функции $f$ имеем

$$
x^{\prime} \in \partial f(x) \Longleftrightarrow f(x)+f^{*}\left(x^{\prime}\right)=\left\langle x, x^{\prime}\right\rangle .
$$

Из теоремы Фенхеля-Моро следует, что если $f$ - собственная полунепрерывная снизу выпуклая функция, то сопряженной к $f^{*}$ является $f$, т.е.

$$
f(x)=\sup _{x^{\prime} \in X^{\prime}}\left[\left\langle x, x^{\prime}\right\rangle-f^{*}\left(x^{\prime}\right)\right], \quad x \in X .
$$

Индикаторной функцией множества $C \subseteq X$ в выпуклом анализе принято называть функцию

$$
\delta_{C}(x)=\left\{\begin{array}{lll}
0, & \text { если } & x \in X \\
+\infty, & \text { если } & x \notin X .
\end{array}\right.
$$

Опорной функцией множества $C$ называется $\delta_{C}^{*}$. Опорные функции множества и его выпуклого замыкания (т.е. замыкания выпуклой оболочки) совпадают. Через int $C$ обозначается множество всех внутренних. точек множества $C$.

Пусть $(\Omega, \mathscr{F})$ - измеримое пространство. Через $\mathscr{L}^{\infty}=\mathscr{L}^{\infty}(\mathscr{F})$ обозначается банахово пространство всех ограниченных измеримых функций $\xi$ с нормой $\|\xi\|=\sup _{\omega}|\xi(\omega)|$. Как известно, сопряженное к $\mathscr{L}^{\infty}$ пространство можно отождествить с пространством $b a=b a(\mathscr{F})$ всех конечно-аддитивных ограниченных функций $\mu: \mathscr{F} \rightarrow \mathbf{R}$ с нормой полной вариации

$$
\|\mu\|=\sup \sum_{i=1}^{n}\left|\mu\left(A_{i}\right)\right|,
$$

где супремум берется по всем конечным разбиениям $\Omega$ на дизъюнктные множества $A_{1}, \ldots, A_{n} \in \mathscr{F}$. Двойственность $\mathscr{L}^{\infty}$ и $b a$ задается соотношением

$$
\langle\xi, \mu\rangle=\mu(\xi):=\int_{\Omega} \xi d \mu, \quad \xi \in \mathscr{L}^{\infty}, \quad \mu \in b a .
$$


Подпространство пространства $b a$, состоящее из всех счетно-аддитивных (знакопеременных) мер, обозначается $c a$. Для $\mu \in b a$ существует единственное разложение $\mu=\mu^{c}+\mu^{a}$, где $\mu^{c} \in c a$, а $\mu^{a}$ - так называемая чисто конечно-аддитивная мера, см., например, [30]. Если $\mu$ принимает неотрицательные значения, то тем же свойством обладают $\mu^{c}$ и $\mu^{a}$.

Пусть $\mathrm{P}$ - вероятностная мера на $(\Omega, \mathscr{F})$. Банаховы пространства $L^{1}(\mathrm{P})=L^{1}(\mathscr{F}, \mathrm{P})$ и $L^{\infty}(\mathrm{P})=L^{\infty}(\mathscr{F}, \mathrm{P})$ понимаются в обычном смысле. Положим $b a(\mathrm{P}):=\{\mu \in b a: \mu(A)=0$ для всех $A \in \mathscr{F}$ с $\mathrm{P}(A)=0\}$, $c a(\mathrm{P})=b a(\mathrm{P}) \cap c a$. Пространство $c a(\mathrm{P})$ можно отождествить с $L^{1}(\mathrm{P})$, a $b a(\mathrm{P})-$ с сопряженным к $L^{\infty}(\mathrm{P})$. Если $\mu \in b a(\mathrm{P})$, то $\mu^{c}, \mu^{a} \in b a(\mathrm{P})$. Сопряженное к банахову пространству $c a$ (с нормой полной вариации) обозначается $c a^{*}$.

Нижний индекс + у рассматриваемых функциональных пространств указывает на конус неотрицательных элементов (элементов с неотрицательными значениями).

Везде используются соглашения $0 \cdot(+\infty)=0$ и $a \cdot(+\infty)=+\infty$ при $a>0$.

2. Определение $f$-дивергенции конечно-аддитивных мер и ее основные свойства. Пусть $f: \mathbf{R} \rightarrow \mathbf{R} \cup\{+\infty\}-$ собственная полунепрерывная снизу выпуклая функция с $\operatorname{dom} f \subseteq \mathbf{R}_{+}$. По функции $f$ построим функции $g$ и $F$, определенные на $\mathbf{R}$ и $\mathbf{R}^{2}$ соответственно, со значениями в $\mathbf{R} \cup\{+\infty\}$ :

$$
\begin{gathered}
g(x)=\sup _{y \in \mathbf{R}}[x y-f(y)], \quad x \in \mathbf{R}, \\
F(s, t)=\sup _{x, y \in \mathbf{R}: y+g(x) \leqslant 0}(s x+t y), \quad(s, t) \in \mathbf{R}^{2} .
\end{gathered}
$$

Свойства функций $g$ и $F$ будут изучаться в п. 4. В частности, будет показано, что $g$ и $F-$ собственные полунепрерывные снизу выпуклые функции, предел

$$
\frac{f(\infty)}{\infty}:=\lim _{y \uparrow+\infty} \frac{f(y)}{y}
$$

существует и совпадает с $\sup \{x: x \in \operatorname{dom} g\}$,

$$
F(s, t)=\left\{\begin{array}{llll}
t f\left(\frac{s}{t}\right), & \text { если } s \geqslant 0 \quad \text { и } t>0, \\
s \frac{f(\infty)}{\infty}, & \text { если } s>0 \text { и } t=0, \\
0, & \text { если } s=0 \text { и } t=0, \\
+\infty, & \text { если } s<0 \text { или } t<0
\end{array}\right.
$$

(ср. с (1)); в частности, функция $F$ является положительно-однородной и $f(y)=F(y, 1)$ для всех $y \in \mathbf{R}$. 
О п р е д е л е н и е $1 . \quad f$-дивергенцией конечно-аддитивных функций множества $\mu$ и $\nu$, заданных на общем измеримом пространстве $(\Omega, \mathscr{F})$, называется величина

$$
\mathscr{J}_{f}(\mu, \nu)=\sup _{\xi, \eta \in \mathscr{L} \infty(\mathscr{F}): \eta+g(\xi) \leqslant 0}[\mu(\xi)+\nu(\eta)]
$$

Основные свойства так определенной $f$-дивергенции приведены в следующей теореме.

Tеорема 1. (i) Функиия $\mathscr{J}_{f}(\mu, \nu)$ на ba $\times$ ba принимает значения в $\mathbf{R} \cup\{+\infty\}$, выпукла и полунепрерывна снизу в топологии $\sigma(b a \times$ ba, $\left.\mathscr{L}^{\infty} \times \mathscr{L}^{\infty}\right)$.

(ii) Если $\mathscr{G}-\sigma$-алгебра, $\mathscr{G} \subseteq \mathscr{F}, \operatorname{mo}_{f}\left(\left.\mu\right|_{\mathscr{G}},\left.\nu\right|_{\mathscr{G}}\right) \leqslant \mathscr{J}_{f}(\mu, \nu)$.

(iii) Для любых $\mu, \nu \in b a$

$$
\mathscr{J}_{f}(\mu, \nu)=\sup \sum_{i=1}^{n} F\left(\mu\left(A_{i}\right), \nu\left(A_{i}\right)\right),
$$

әде супремум берется по всем конечным разбиениям $\Omega$ на дизъюнктные множества $A_{1}, \ldots, A_{n} \in \mathscr{F}$. В частности, если $\mu \notin b a_{+}$или $\nu \notin b a_{+}$, mo $\mathscr{J}_{f}(\mu, \nu)=+\infty$.

(iv) Для любых $\mu, \nu \in b a$

$$
\mathscr{J}_{f}(\mu, \nu)=\mathscr{J}_{f}\left(\mu^{c}, \nu^{c}\right)+\mathscr{J}_{f}\left(\mu^{a}, \nu^{a}\right) \text {. }
$$

(v) Для любых $\mu, \nu \in$ са $и$ б-конечной неотрицательной меры $\varrho$ на $(\Omega, \mathscr{F})$, доминируюшей $\mu$ и,

$$
\mathscr{J}_{f}(\mu, \nu)=\int_{\Omega} F\left(\frac{d \mu}{d \varrho}, \frac{d \nu}{d \varrho}\right) d \varrho .
$$

(vi) Для любых $\mu, \nu \in b a_{+}$

$$
\mathscr{J}_{f}(\mu, \nu)=\sup _{\xi \in \mathscr{L} \infty: g(\xi) \in \mathscr{L} \infty}[\mu(\xi)-\nu(g(\xi))]
$$

(vii) Для любых $\mu, \nu \in b a_{+}$

$$
F(\mu(\Omega), \nu(\Omega)) \leqslant \mathscr{J}_{f}(\mu, \nu) \leqslant \frac{f(\infty)}{\infty} \mu(\Omega)+f(0) \nu(\Omega) .
$$

(viii) Для любой $\mu \in b a_{+}$

$$
\mathscr{J}_{f}(\mu, 0)=\frac{f(\infty)}{\infty} \mu(\Omega), \quad \mathscr{J}_{f}(0, \mu)=f(0) \mu(\Omega) .
$$

(ix) Ecлut, s>0, mo $\mathscr{J}_{f}(t \mu, t \nu)=t \mathscr{J}_{f}(\mu, \nu) u \mathscr{J}_{f}(t \mu, s \nu)=\mathscr{J}_{h}(\mu, \nu)$ для любьх $\mu, \nu \in b a$, где $h(y)=s f(t y / s), y \in \mathbf{R}$. 
(х) Если $f_{1}$ и $f_{2}$ - собственнье полунепрерьъвнье снизу выпукльге бункиии с $\operatorname{dom} f_{i} \subseteq \mathbf{R}_{+}, i=1,2, u \operatorname{dom} f_{1} \cap \operatorname{dom} f_{2} \neq \varnothing$, mo

$$
\mathscr{J}_{f_{1}+f_{2}}(\mu, \nu)=\mathscr{J}_{f_{1}}(\mu, \nu)+\mathscr{J}_{f_{2}}(\mu, \nu)
$$

для любых $\mu, \nu \in b a$.

(xi) Ecsu $f(y)=t y+s, y \in \mathbf{R}_{+}, m o \mathscr{J}_{f}(\mu, \nu)=t \mu(\Omega)+s \nu(\Omega) \partial \Omega g$ любьљх $\mu, \nu \in b a_{+}$.

(xii) Пусть $\operatorname{dom} f \neq\{0\}$. Положим $\hat{f}(y)=F(1, y), y \in \mathbf{R}$. Тогда $\hat{f}-$ собственная полунепрерьвная снизу выпуклая функция $c \operatorname{dom} \hat{f} \subseteq \mathbf{R}_{+} u \mathscr{J}_{f}(\nu, \mu)=\mathscr{J}_{\hat{f}}(\mu, \nu)$ для $л ю б ъ х x ~ \mu, \nu \in b a$.

Как отмечалось выше, в классическом случае $(f$ конечна на $(0,+\infty)$, меры счетно-аддитивны) в качестве определения $f$-дивергенции принимается соотношение (9); утверждение (v) показывает, что наше определение 1 согласуется с классическим. То же следует из утверждения (iii): в классическом случае соотношение (7) хорошо известно (см. [23, следствие 1.32]); более того, супремум можно брать по всем разбиениям $\Omega$ на множества из фиксированной алгебры $\mathscr{A}$, порождающей $\sigma$-алгебру $\mathscr{F}$. Для конечно-аддитивных мер это уже не так, поскольку продолжение конечно-аддитивной меры с алгебры на порождающую ее $\sigma$-алгебру, вообще говоря, неединственно. Монотонность (ii) является одним из основных свойств $f$-дивергенции, в классическом случае см. [23, теорема 1.24 и следствие 1.29]. Более тонкий результат, касающийся полунепрерывности снизу $f$-дивергенции в классическом случае, см. в [23, теорема 1.47]. По поводу области значений $f$-дивергенции (утверждение (vii)) и свойства симметрии (утверждение (хіi)) в классическом случае см. [23, теоремы 1.12 и 1.13$]$.

В заметке [17] мы определили $f$-дивергенцию конечно-аддитивных мер соотношением (7). Здесь мы предпочитаем взять определение 1 за исходное в силу соображений методологического характера. Отметим, например, что утверждения (i) и (ii) теоремы 1 непосредственно вытекают из определения 1 , а утверждение (v) и частично утверждение (iv) суть немедленные следствия определения 1 и результатов Р. Рокафеллара [25], [26] о виде сопряженного функционала к функционалу интегрального типа. Действительно, пусть $\mu, \nu \in b a$ и существует такая вероятностная мера $\mathrm{P}$, для которой $\mu, \nu \in b a(\mathrm{P})$ (что не ограничивает общности, если $\mu$ и $\nu$ счетно-аддитивны). Определим на $L^{\infty}(\mathrm{P}) \times L^{\infty}(\mathrm{P})$ функционал

$$
N(\xi, \eta)=\int_{\Omega} \delta_{D}(\xi, \eta) d \mathrm{P}
$$

где $D=\left\{(x, y) \in \mathbf{R}^{2}: y+g(x) \leqslant 0\right\}$. Легко видеть, что в рассматриваемом случае определение 1 сводится к равенству $\mathscr{J}_{f}(\mu, \nu)=N^{*}(\mu, \nu)$. 
С другой стороны, сопряженной к функции $\delta_{D}$ является $F$ в силу определения (5). Поэтому из теоремы 2 в [25] и теоремы 1 в [26] следует, что

$$
\begin{aligned}
N^{*}(\mu, \nu) & =\int_{\Omega} F\left(\frac{d \mu^{c}}{d \mathrm{P}}, \frac{d \nu^{c}}{d \mathrm{P}}\right) d \mathrm{P}+\sup _{\substack{\xi, \eta \in L^{\infty}(\mathrm{P}): \\
N(\xi, \eta)<+\infty}}\left[\mu^{a}(\xi)+\nu^{a}(\eta)\right] \\
& =\mathscr{J}_{f}\left(\mu^{c}, \nu^{c}\right)+\mathscr{J}_{f}\left(\mu^{a}, \nu^{a}\right) .
\end{aligned}
$$

Для полноты изложения мы приведем в п. 5 другое доказательство утверждения (v). Что касается утверждения (iv), то оно будет доказано в полном объеме также в п. 5, но при этом ход рассуждений будет следовать доказательству теоремы 1 в [26].

В заключение отметим, что если функция $\mathscr{J}(\mu, \nu)$ на $b a \times b a$ полунепрерывна снизу в топологии $\sigma\left(b a \times b a, \mathscr{L}^{\infty} \times \mathscr{L}^{\infty}\right)$ и совпадает с $\mathscr{J}_{f}(\mu, \nu)$ для $\mu, \nu \in c a_{+}$(или хотя бы для пар $(\mu, \nu)$ специального вида, см. ниже), то $\mathscr{J}(\mu, \nu) \leqslant \mathscr{J}_{f}(\mu, \nu)$ на $b a \times b a$. Это вытекает из следуюшего рассуждения. Пусть $\mu, \nu \in b a_{+}$, а $\lambda$ - конечное разбиение $\Omega$ на непустые дизъюнктные множества $A_{1}, \ldots, A_{n} \in \mathscr{F}$. Для каждого $i=1, \ldots, n$ зафиксируем точку $\omega_{i} \in A_{i}$ и положим $\mu_{\lambda}=\sum_{i=1}^{n} \mu\left(A_{i}\right) \varepsilon_{\omega_{i}}, \nu_{\lambda}=\sum_{i=1}^{n} \nu\left(A_{i}\right) \varepsilon_{\omega_{i}}$, где $\varepsilon_{\omega}$ - мера Дирака в точке $\omega$. Упорядочим разбиения $\lambda$ по вложению порожденных ими конечных $\sigma$-алгебр $\sigma\{\lambda\}$. Легко видеть, что направленность (обобщенная последовательность, сеть) $\left(\mu_{\lambda}, \nu_{\lambda}\right)$ сходится к $(\mu, \nu)$ в топологии $\sigma\left(b a \times b a, \mathscr{L}^{\infty} \times \mathscr{L}^{\infty}\right)$. При этом в силу (9)

$$
\mathscr{J}_{f}\left(\mu_{\lambda}, \nu_{\lambda}\right)=\mathscr{J}_{f}\left(\left.\mu\right|_{\sigma\{\lambda\}},\left.\nu\right|_{\sigma\{\lambda\}}\right)=\sum_{i=1}^{n} F\left(\mu\left(A_{i}\right), \nu\left(A_{i}\right)\right),
$$

и из утверждений (ii), (iii) теоремы 1 следует, что

$$
\mathscr{J}_{f}(\mu, \nu)=\lim _{\lambda} \mathscr{J}_{f}\left(\mu_{\lambda}, \nu_{\lambda}\right) .
$$

3. Применение к задаче минимизации $f$-дивергенции. Пусть $\mathbb{P}$ - множество всех вероятностных мер на измеримом пространстве $(\Omega, \mathscr{F}), f: \mathbf{R} \rightarrow \mathbf{R} \cup\{+\infty\}-$ собственная полунепрерывная снизу выпуклая функция с $\operatorname{dom} f \subseteq \mathbf{R}_{+}$. Предположим, что задано множество $\mathscr{Z} \subseteq \mathbb{P} \times \mathbb{P}$. В этом пункте мы рассматриваем одну частную постановку следующего общего вопроса: при каких предположениях (на $f$ и $\mathscr{Z}$ ) $f$-дивергенция $\mathscr{J}_{f}(\mathrm{P}, \mathrm{Q})$ достигает своего минимума на $\mathscr{Z}$, т.е. существует экстремальная пара $\left(\mathrm{P}_{*}, \mathrm{Q}_{*}\right) \in \mathscr{Z}$, для которой

$$
\mathscr{J}_{f}\left(\mathrm{P}_{*}, \mathrm{Q}_{*}\right) \leqslant \mathscr{J}_{f}(\mathrm{P}, \mathrm{Q}) \quad \text { для всех }(\mathrm{P}, \mathrm{Q}) \in \mathscr{Z} ?
$$

В других постановках задача рассматривалась, например, в работах [8], [28], [23, раздел 8], [7], [21], [4], где можно найти дополнительные ссылки. 
Подчеркнем, что функция $f$ предполагается фиксированной. Мы не рассматриваем более специальный вопрос: когда существует «наименее благоприятная» пара $\left(\mathrm{P}_{*}, \mathrm{Q}_{*}\right) \in \mathscr{Z}$, удовлетворяющая (10) одновременно $\partial л я$ всех $f$ ? Иными словами, когда среди бинарных статистических экспериментов $(\Omega, \mathscr{F},(\mathrm{P}, \mathrm{Q})),(\mathrm{P}, \mathrm{Q}) \in \mathscr{Z}$, существует наименее информативный (в смысле сравнения статистических экспериментов)? В задаче робастной проверки гипотез такой вопрос возникает с множествами $\mathscr{Z}$ вида $\mathscr{P} \times \mathscr{Q} ;$ условия существования наименее информативного эксперимента изучались, например, в работах [19], [2], [20].

При некоторых предположениях интересующий нас вопрос является элементарным, например, если $\mathscr{Z}$ содержит диагональный элемент (P, P). Другое предположение, при котором мы немедленно получаем желаемый ответ, состоит в компактности множества $\mathscr{Z}$ в слабой топологии $\sigma\left(c a \times c a, \mathscr{L}^{\infty} \times \mathscr{L}^{\infty}\right)$. Действительно, в силу утверждения (i) теоремы $1 f$-дивергенция полунепрерывна снизу в (индуцируемой на $c a \times c a$ топологией $\left.\sigma\left(b a \times b a, \mathscr{L}^{\infty} \times \mathscr{L}^{\infty}\right)\right)$ топологии $\sigma\left(c a \times c a, \mathscr{L}^{\infty} \times \mathscr{L}^{\infty}\right)$, а полунепрерывная снизу функция достигает на компакте своего минимума.

В дальнейшем нас будет интересовать, как можно ослабить предположение о компактности $\mathscr{Z}$ при дополнительном ограничении на функцию $f$, а именно, в случае $f(\infty) / \infty=+\infty$. Основные результаты сформулированы ниже в теореме 2 и следствии 2. Приведем рассуждение, на котором основано доказательство теоремы 2. Рассмотрим $\mathscr{Z}$ как подмножество $b a \times b a$, и пусть $\overline{\mathscr{Z}}$ - его замыкание в топологии $\sigma\left(b a \times b a, \mathscr{L}^{\infty} \times \mathscr{L}^{\infty}\right)$. Так как множество $\overline{\mathscr{Z}}$ ограничено по норме, оно компактно в топологии $\sigma\left(b a \times b a, \mathscr{L}^{\infty} \times \mathscr{L}^{\infty}\right)$ по теореме БанахаАлаоглу. Поэтому $f$-дивергенция $\mathscr{J}_{f}(\mu, \nu)$ достигает своего минимума на $\overline{\mathscr{Z}}$, и для того, чтобы она достигала своего минимума на $\mathscr{Z}$, достаточно, чтобы $\mathscr{J}_{f}(\mu, \nu)=+\infty$ для всех $(\mu, \nu) \in \overline{\mathscr{Z}} \backslash \mathscr{Z}$. Последнее будет выполнено, если для любой пары $(\mu, \nu) \in \overline{\mathscr{Z}} \backslash \mathscr{Z}$ имеет место $\mu \notin c a$, $\nu \in c a$ - действительно, тогда $\mu^{a} \neq 0, \nu^{a}=0$, и $\mathscr{J}_{f}(\mu, \nu)=+\infty$ в силу утверждений (iv) и (viii) теоремы 1.

В следующих утверждениях обсуждаются предположения, которые встретятся в теореме 2 и следствии 2.

Предложение 1. Выпуклое множество $\mathscr{P} \subseteq \mathbb{P}$ замкнуто в са относительно топологии $\sigma\left(c a, c a^{*}\right)$ тогда и только тогда, когда оно замкнуто в са относительно сходимости по вариаиии. Выпуклое множество $\mathscr{Z} \subseteq \mathbb{P} \times \mathbb{P}$ замкнуто в са $\times$ са относительно топологии $\sigma\left(c a \times c a, c a^{*} \times c a^{*}\right)$ тогда и только тогда, когда оно замкнуто в са $\times$ са относительно покоординатной сходимости по вариачии.

Д о к а з а т е л с т в о. В банаховом пространстве замкнутое по норме выпуклое множество замкнуто и в слабой топологии. 
Следующее предложение хорошо известно (см., например, [3, теорема 4.7.26], а также [24, раздел IV.2]).

Предложение 2. Пусть $\mathscr{Q} \subseteq \mathbb{P}$. Следуюшие утверждения эквивалентны:

(i) $\mathscr{Q}$ относительно компактно в са относительно топологии $\sigma\left(c a, c a^{*}\right)$;

(ii) $\mathscr{Q}$ относительно компактно в са относительно топологии $\sigma\left(c a, \mathscr{L}^{\infty}\right)$;

(iii) существует такая вероятность $\mathrm{R} \in \mathbb{P}$, ито $\mathrm{Q} \ll \mathrm{R}$ для любой $\mathrm{Q} \in \mathscr{Q}$ и семейство случайньх величин

$$
\left\{\frac{d \mathrm{Q}}{d \mathrm{R}}: Q \in \mathscr{Q}\right\}
$$

равномерно интегрируемо по мере $\mathrm{R}$.

Следствие 1. Пусть $\mathscr{Q} \subseteq \mathbb{P}$. Следуюшие утверждения эквивалентны:

(i) $\mathscr{Q}$ компактно в са относительно топологии $\sigma\left(c a, \mathrm{ca}^{*}\right)$;

(ii) $\mathscr{Q}$ замкнуто в ьа относительно топологии $\sigma\left(b a, \mathscr{L}^{\infty}\right)$.

Более того, если $\mathscr{Q}$ - выпуклое множество, то утверждения (i) и (ii) эквивалентны каждому из следующих утверждений:

(iii) существует такая вероятность $\mathrm{R} \in \mathbb{P}$, что $\mathrm{Q} \ll \mathrm{R}$ для любой $\mathrm{Q} \in \mathscr{Q}$ и семейство случайньх величин

$$
\left\{\frac{d \mathrm{Q}}{d \mathrm{R}}: \mathrm{Q} \in \mathscr{Q}\right\}
$$

замкнуто в $L^{1}(\mathrm{R})$ и равномерно интегрируемо по мере $\mathrm{R}$;

(iv) существует такая вероятность $\mathrm{R} \in \mathbb{P}$, ито $\mathrm{Q} \ll \mathrm{R}$ для любой $\mathrm{Q} \in \mathscr{Q}$ и семейство случайньх величин

$$
\left\{\frac{d \mathrm{Q}}{d \mathrm{R}}: \mathrm{Q} \in \mathscr{Q}\right\}
$$

замкнуто относительно сходимости по мере $\mathrm{R}$.

Д о к а з а т ел ь с т в о. Так как элементы $\mathscr{Q}$ принадлежат единичному шару в $b a$, по теореме Банаха-Алаоглу утверждение (ii) эквивалентно компактности $\mathscr{Q}$ в $b a$ относительно топологии $\sigma\left(b a, \mathscr{L}^{\infty}\right)$, что эквивалентно компактности $\mathscr{Q}$ в са относительно топологии $\sigma\left(c a, \mathscr{L}^{\infty}\right)$. Так как топология $\sigma\left(c a, c a^{*}\right)$ сильнее, чем топология $\sigma\left(c a, \mathscr{L}^{\infty}\right)$, из (i) следует (ii). По той же причине из (ii) следует замкнутость $\mathscr{Q}$ в $с а$ относительно топологии $\sigma\left(c a, c a^{*}\right)$, и импликация (ii) $\Rightarrow(\mathrm{i})$ следует из аналогичной импликации в предложении 2. Даже без предположения о выпуклости $\mathscr{Q}$ импликация (iii) $\Rightarrow$ (iv) тривиальна, a (i) $\Rightarrow$ (iii) немедленно следует из аналогичной импликации в предложении 2. 
Что касается импликации (iv) $\Rightarrow$ (i), то ее короткий вывод можно найти в [29, лемма 3.2]; однако этот вывод основан на глубоком результате из общей теории банаховых пространств - теореме Джеймса о компактных множествах в слабой топологии. Другое доказательство можно найти в [3, пример 4.7.25]. Для удобства читателя мы сформулируем и докажем лемму 1, которая немедленно дает еще одно доказательство импликации (iv) $\Rightarrow$ (ii). Следствие 1 доказано.

Лемма 1. Предположим, что $\mathrm{R}$ - вероятностная мера, множество $M \subseteq L^{1}(\mathrm{R})$ состоит из вероятностньх плотностей $u$ выпукло. Пусть $\bar{M}$ - замькание $M$ относительно сходимости по мере $\mathrm{R}$, $\mathscr{Q}=\{\mathrm{Q} \in \mathbb{P}: d \mathrm{Q} / d \mathrm{R} \in M\}, \overline{\mathscr{Q}}$ - замькание $\mathscr{Q}$ в ьа относительно топологии $\sigma\left(b a, \mathscr{L}^{\infty}\right)$. Тогда

$$
\left\{\frac{d \mu^{c}}{d \mathrm{R}}: \mu \in \overline{\mathscr{Q}}\right\}=\bar{M} .
$$

3 а м е ч а н и я. 1. Ясно, что $\overline{\mathscr{Q}}$ есть также замыкание $\mathscr{Q}$ в $b a(\mathrm{R})$ относительно топологии $\sigma\left(b a(\mathrm{R}), L^{\infty}(\mathrm{R})\right)$. В частности, $\mu^{c} \ll \mathrm{R}$ для любой $\mu \in \overline{\mathscr{Q}}$.

2. Очевидно, что множество $\bar{M}$ можно также определить как множество пределов всех последовательностей из $M$, сходящихся R-п.н.

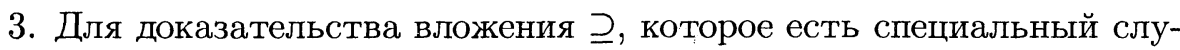
чай предложения А.1 в [9], выпуклость $\mathscr{Q}$ не используется.

Д о к а з а т ел ь с т в о. Если $\mu \in b a, A \in \mathscr{F}$, то $\left.\mu\right|_{A}$ определяется соотношением $\left.\mu\right|_{A}(B)=\mu(B \cap A), B \in \mathscr{F}$.

() Пусть $\left\{\xi_{n}\right\}$ - последовательность из $M$, сходящаяся R-п.н. к $\xi$, а меры $\mathrm{Q}_{n} \in \mathscr{Q}$ определены соотношением $d \mathrm{Q}_{n} / d \mathrm{R}=\xi_{n}$. По теореме Банаха-Алаоглу последовательность $\left\{Q_{n}\right\}$ имеет в $b a$ предельную точку относительно топологии $\sigma\left(b a, \mathscr{L}^{\infty}\right)$, скажем, $\mu$. Покажем, что $d \mu^{c} / d \mathrm{R}=\xi \mathrm{R}$-п.н. По теореме Егорова для любого натурального $k$ найдется такое множество $A_{k} \in \mathscr{F}$, что $\mathrm{R}\left(A_{k}\right)>1-1 / k$ и $\xi_{n}$ сходятся к $\xi$ равномерно на $A_{k}$ при $n \rightarrow \infty$. В частности, $\xi_{n} \mathbf{1}_{A_{k}}$ сходятся к $\xi \mathbf{1}_{A_{k}}$ в $L^{1}(\mathrm{R})$ для каждого $k$, т.е. последовательность $\left\{\left.\mathrm{Q}_{n}\right|_{A_{k}}\right\}$ сходится по вариации к счетно-аддитивной мере с плотностью $\xi 1_{A_{k}}$ относительно R. Но $\left.\mu\right|_{A_{k}}$ есть предельная точка последовательности $\left\{\left.\mathrm{Q}_{n}\right|_{A_{k}}\right\}$ относительно топологии $\sigma\left(b a, \mathscr{L}^{\infty}\right)$. Значит, $\left.\mu\right|_{A_{k}}=\left.\mu^{c}\right|_{A_{k}}$ и $\left(d \mu^{c} / d \mathrm{R}\right) \mathbf{1}_{A_{k}}=\xi \mathbf{1}_{A_{k}}$ для любого $k$, откуда $d \mu^{c} / d \mathrm{R}=\xi \mathrm{R}$-п.н.

$(\subseteq)$ Пусть $\mu \in \overline{\mathscr{Q}} \backslash \mathscr{Q} \subseteq b a_{+}$. По известному свойству чисто конечноаддитивных мер (см. [30, теорема 1.19]), найдется такая последовательность $\left\{A_{k}\right\}$ множеств из $\mathscr{F}$, что $\mathrm{R}\left(A_{k}\right)>1-1 / k$ и $\mu^{a}\left(A_{k}\right)=0$ для всех $k$; переходя к конечным объединениям, можно считать последовательность $\left\{A_{k}\right\}$ возрастающей. Мера $\left.\mu\right|_{A_{k}}$ счетно-аддитивна; кроме того, она принадлежит замыканию выпуклого множества $\left\{\left.Q\right|_{A_{k}}: Q \in \mathscr{Q}\right\}$, 
причем замыкание можно брать в пространстве $c a(\mathrm{R})$ относительно топологии $\sigma\left(c a(\mathrm{R}), L^{\infty}(\mathrm{R})\right)$. Эквивалентно, $\xi \mathbf{1}_{A_{k}}$, где $\xi=d \mu^{c} / d \mathrm{R}$, лежит в замыкании выпуклого множества $\left\{\zeta \mathbf{1}_{A_{k}}: \zeta \in M\right\}$ в $L^{1}(\mathrm{R})$ относительно топологии $\sigma\left(L^{1}(\mathrm{R}), L^{\infty}(\mathrm{R})\right)$. Так как замыкания выпуклого множества в сильной и слабой топологиях банахова пространства совпадают, для любого $k$ найдется такая $\zeta_{k} \in M$, что $\left\|\left(\xi-\zeta_{k}\right) \mathbf{1}_{A_{k}}\right\|_{L^{1}(\mathrm{R})}<1 / k$. Построим такие выпуклые комбинации $\eta_{k} \in \operatorname{conv}\left\{\zeta_{k}, \zeta_{k+1}, \ldots\right\} \subseteq M$, что $\eta_{k}$ сходятся R-п.н. к некоторой $\eta \in \bar{M}$ при $k \rightarrow \infty$ (это можно сделать, например, в силу леммы А1.1 в [10]). Тогда при $n \geqslant k$ имеем $\left\|\left(\xi-\zeta_{n}\right) \mathbf{1}_{A_{k}}\right\|_{L^{1}(\mathrm{R})} \leqslant$ $\left\|\left(\xi-\zeta_{n}\right) \mathbf{1}_{A_{n}}\right\|_{L^{1}(\mathrm{R})}<1 / n \leqslant 1 / k$, поэтому $\left\|\left(\xi-\eta_{k}\right) \mathbf{1}_{A_{k}}\right\|_{L^{1}(\mathrm{R})}<1 / k$, откуда $\left(\xi-\eta_{k}\right) \mathbf{1}_{A_{k}} \stackrel{\mathrm{R}}{\longrightarrow} 0$; значит, $\xi=\eta \mathrm{R}$-п.н. Лемма 1 доказана.

Теорема 2. Пусть $f(\infty) / \infty=+\infty$, множество $\mathscr{Z} \subseteq \mathbb{P} \times \mathbb{P}$ замкнуто в са $\times$ са относительно топологии $\sigma\left(c a \times c a, c a^{*} \times c a^{*}\right)$, а проекиия $\mathscr{Q}:=\{\mathrm{Q} \in \mathbb{P}:(\mathrm{P}, \mathrm{Q}) \in \mathscr{Z}$ для некоторой $\mathrm{P} \in \mathbb{P}\}$ относительно компактна в са относительно топологии $\sigma\left(c a, c a^{*}\right)$. Тогда найдется maкая пара $\left(\mathrm{P}_{*}, \mathrm{Q}_{*}\right) \in \mathscr{Z}$, чmo

$$
\mathscr{J}_{f}\left(\mathrm{P}_{*}, \mathrm{Q}_{*}\right) \leqslant \mathscr{J}_{f}(\mathrm{P}, \mathrm{Q}) \quad \text { для всех }(\mathrm{P}, \mathrm{Q}) \in \mathscr{Z} .
$$

Следующий результат есть частный случай теоремы 2.

Следствие 2. Пусть $f(\infty) / \infty=+\infty$, множество $\mathscr{P} \subseteq \mathbb{P}$ замкнуто в са относительно топологии $\sigma\left(c a, c a^{*}\right)$, а множество $\mathscr{Q} \subseteq \mathbb{P}$ компактно в са относительно топологии $\sigma\left(c a, c^{*}\right)$. Тогда найдутся такие мерь $\mathrm{P}_{*} \in \mathscr{P} u \mathrm{Q}_{*} \in \mathscr{Q}$, ито

$$
\mathscr{J}_{f}\left(\mathrm{P}_{*}, \mathrm{Q}_{*}\right) \leqslant \mathscr{J}_{f}(\mathrm{P}, \mathrm{Q}) \quad \text { для всех } \mathrm{P} \in \mathscr{P}, \mathrm{Q} \in \mathscr{Q} .
$$

При классических предположениях на $f$ и для выпуклых $\mathscr{P}, \mathscr{Q}$ следствие 2 не является новым. Если множество $\mathscr{P}$ выпукло, а $\mathscr{Q}=\{Q\}$ состоит из одной точки, утверждение было доказано И. Чисаром [6] в случае $f(u)=u \ln u$ и $\Phi$. Лизе [22] в общем случае (см. также [23, предложение 8.5]). Для выпуклых доминируемых $\mathscr{P}$ и $\mathscr{Q}$ следствие 2 было недавно доказано Х. Фёльмером и А. Гундель [12] довольно трудоемким методом.

Д ок азат е л ь с т в о т е о р е м ы 2. В силу предложения 2 семейство $\mathscr{Q}$ доминируется некоторой вероятностной мерой R. Если мера $\mathrm{P} \in \mathbb{P}$ не абсолютно непрерывна относительно $\mathrm{R}$, то $\mathscr{J}_{f}(\mathrm{P}, \mathrm{Q})=+\infty$ для любой $Q \in \mathscr{Q}$ в силу утверждения (iii) теоремы 1 . Заметим, что $c a(\mathrm{R})$ замкнуто в $c a$ относительно топологии $\sigma\left(c a, c a^{*}\right)$. Поэтому, заменяя множество $\mathscr{Z}$ на $\{(\mathrm{P}, \mathrm{Q}) \in \mathscr{Z}: \mathrm{P} \ll \mathrm{R}\}$, можно считать без ограничения общности, что $\mathrm{P} \ll \mathrm{R}$, если $(\mathrm{P}, \mathrm{Q}) \in \mathscr{Z}$. Пусть $\overline{\mathscr{Z}}-$ замыкание $\mathscr{Z}$ в $b a \times b a$ относительно топологии $\sigma\left(b a \times b a, \mathscr{L}^{\infty} \times \mathscr{L}^{\infty}\right)$, и пусть $(\mu, \nu) \in \overline{\mathscr{Z}} \backslash \mathscr{Z}$. Тогда $\nu$ лежит в замыкании множества $\mathscr{Q}$ в bа относительно топологии $\sigma\left(b a, \mathscr{L}^{\infty}\right)$. Из предположения на $\mathscr{Q}$ и предложения 2 
следует, что замыкание $\overline{\mathscr{Q}}$ множества $\mathscr{Q}$ в са относительно топологии $\sigma\left(c a, \mathscr{L}^{\infty}\right)$ компактно. Так как топология $\sigma\left(c a, \mathscr{L}^{\infty}\right)$ индуцируется топологией $\sigma\left(b a, \mathscr{L}^{\infty}\right)$, множество $\overline{\mathscr{Q}}$ также компактно в $b a$ относительно топологии $\sigma\left(b a, \mathscr{L}^{\infty}\right) ;$ в частности, $\nu \in \overline{\mathscr{Q}} \subseteq c a . \quad$ В силу приведенного в начале этого пункта рассуждения остается показать, что $\mu \notin c a$. Отождествим знакопеременные счетно-аддитивные меры, абсолютно непрерывные относительно $\mathrm{R}$, с элементами $L^{1}(\mathrm{R})$. Поскольку топология на $L^{1}(\mathrm{R}) \times L^{1}(\mathrm{R})$, индуцируемая топологией $\sigma\left(c a \times c a, c a^{*} \times c a^{*}\right)$, совпадает с топологией $\sigma\left(L^{1}(\mathrm{R}) \times L^{1}(\mathrm{R}), L^{\infty}(\mathrm{R}) \times L^{\infty}(\mathrm{R})\right)$, множество $\mathscr{Z}$ замкнуто в $L^{1}(\mathrm{R}) \times L^{1}(\mathrm{R})$ относительно топологии $\sigma\left(L^{1}(\mathrm{R}) \times L^{1}(\mathrm{R}), L^{\infty}(\mathrm{R}) \times L^{\infty}(\mathrm{R})\right)$ и, следовательно, замкнуто в $c a \times c a$ относительно топологии $\sigma(c a \times$ $\left.c a, \mathscr{L}^{\infty} \times \mathscr{L}^{\infty}\right)$. Поэтому $(\mu, \nu) \notin c a \times c a$, т.е. $\mu \notin c a$. Теорема 2 доказана.

4. Вспомогательные результаты. Обозначим $\mathscr{V}$ класс всех собственных полунепрерывных снизу выпуклых функций $f: \mathbf{R} \rightarrow \mathbf{R} \cup\{+\infty\}$ c $\operatorname{dom} f \subset \mathbf{R}_{+}$, а $\mathscr{W}$ - класс всех собственных полунепрерывных снизу выпуклых неубывающих функций $g: \mathbf{R} \rightarrow \mathbf{R} \cup\{+\infty\}$.

Предложение 3. Отображение $\mathscr{V} \ni f \rightsquigarrow g$, задаваемое соотношением

$$
g(x)=\sup _{y \in \mathbf{R}}[x y-f(y)], \quad x \in \mathbf{R},
$$

является взаимно однозначным отображением из $\mathscr{V}$ на $\mathscr{W}$. Обратное отображение задается формулой

$$
f(y)=\sup _{x \in \mathbf{R}}[x y-g(x)], \quad y \in \mathbf{R} .
$$

Д о к а з а т е л ь с т в о. Пусть $f \in \mathscr{V}$ и $g$ задается формулой (11). Тогда $g$ - преобразование Фенхеля функции $f$, значит, $g$ - собственная полунепрерывная снизу выпуклая функция, и по теореме ФенхеляМоро имеет место (12), в частности, рассматриваемое отображение инъективно. Так как $\operatorname{dom} f \subset \mathbf{R}_{+}$, из (11) следует, что $g$ не убывает, т.е. $g \in \mathscr{W}$.

Пусть теперь $g \in \mathscr{W}$. Зададим функцию $f$ соотношением (12). Тогда $f$ - собственная полунепрерывная снизу выпуклая функция. Поскольку $g$ не убывает, из (12) ясно, что $f(y)=+\infty$ при $y<0$, поэтому $f \in \mathscr{V}$. По теореме Фенхеля-Моро имеет место (11), поэтому образ $\mathscr{V}$ при рассматриваемом отображении совпадает с $\mathscr{W}$. Предложение 3 доказано.

Введем обозначения, используемые далее в этом пункте. Пусть $h: \mathbf{R} \rightarrow \mathbf{R} \cup\{+\infty\}-$ собственная полунепрерывная снизу выпуклая функция. Положим

$$
l_{h}=\inf \{x: x \in \operatorname{dom} h\}, \quad r_{h}=\sup \{x: x \in \operatorname{dom} h\} .
$$


Пусть $l_{h} \leqslant \alpha<\beta \leqslant r_{h}$. Если $\alpha \in \mathbf{R}$ и $h(\alpha) \in \mathbf{R}$, то через $h_{+}^{\prime}(\alpha)$ обозначаем правую производную $h$ в точке $\alpha$, в противном случае $h_{+}^{\prime}(\alpha)$ понимается как $\lim _{x \downarrow \alpha} h_{+}^{\prime}(x)$. Символ $h_{-}^{\prime}(\beta)$ понимается аналогично. Положим

$$
\begin{aligned}
L_{h} & =\left\{\begin{array}{lll}
\varnothing, & \text { если } & l_{h}>-\infty, \\
\left\{x \in \operatorname{dom} h: h_{-}^{\prime}(x)=h_{+}^{\prime}(-\infty)\right\}, & \text { если } & l_{h}=-\infty,
\end{array}\right. \\
R_{h} & =\left\{\begin{array}{lll}
\varnothing, & \text { если } & r_{h}<+\infty \\
\left\{x \in \operatorname{dom} h: h_{+}^{\prime}(x)=h_{-}^{\prime}(+\infty)\right\}, & \text { если } & r_{h}=+\infty
\end{array}\right.
\end{aligned}
$$

Предложение 4. Пусть функиии $f \in \mathscr{V} u g \in \mathscr{W}$ связань соотношениями (11) и (12). Имеют место следуюшие утверждения.

(i) $l_{f}=g_{+}^{\prime}(-\infty) u \partial f\left(l_{f}\right)=L_{g}$.

(ii) $r_{g}=+\infty$ и $g_{-}^{\prime}(+\infty)=+\infty$ тогда и только тогда, когда $r_{f}=+\infty u f_{-}^{\prime}(+\infty)=+\infty$.

(iii) $r_{g}=+\infty$ u $g_{-}^{\prime}(+\infty)<+\infty$ тогда и только тогда, когда $r_{f}<+\infty$. При этом $r_{f}=g_{-}^{\prime}(+\infty)$ и $\partial f\left(r_{f}\right)=R_{g}$.

(iv) $r_{g}<+\infty$ тогда и только тогда, когда $r_{f}=+\infty$ u $f_{-}^{\prime}(+\infty)<$ $+\infty$. При этом $r_{g}=f_{-}^{\prime}(+\infty)$ и $\partial g\left(r_{g}\right)=R_{f}$.

(v) Функиия $g$ диФФерениируема на int $\operatorname{dom} g$ тогда и только тогда, когда функиия $f$ строго выпукла на $\operatorname{dom} f \backslash R_{f}$. Функиия $f$ строго выпукла на $\operatorname{dom} f$ тогда и только тогда, когда функиия $g$ дифференчируема на int dom $g$ и $R_{f}=\varnothing$.

(vi) Функиия $f$ дифферениируема на int $\operatorname{dom} f$ тогда и только тогда, когда функиия $g$ строго выпукла на $\operatorname{dom} g \backslash\left(L_{g} \cup R_{g}\right)$. Функиия $g$ строго выпукла на $\operatorname{dom} g$ тогда и только тогда, когда функиия $f$ дифферениируема на int dom $f$ u $L_{g} \cup R_{g}=\varnothing$.

Доказательство всех утверждений основано на известном соотношении

$$
x \in \partial f(y) \Longleftrightarrow y \in \partial g(x), \quad x, y \in \mathbf{R}
$$

(ср. с (3)), и предоставляется читателю (см. также [27, раздел 26]).

Сформулируем два следствия предложений 3 и 4. В первом из них описывается в терминах $g$ ситуация, когда функция $f$ конечна на $(0,+\infty)$, т.е. принадлежит классу функций, для которых определяется $f$-дивергенция в классическом смысле.

Следствие 3. Пусть функции $f \in \mathscr{V} u g \in \mathscr{W}$ связань соотношениями (11) и (12). Для того чтобы выполнялось включение $\operatorname{dom} f \supseteq(0,+\infty)$, необходимо и достаточно, чтобы $g_{+}^{\prime}(-\infty)=0$ и либо $r_{g}<+\infty$, либо $r_{g}=+\infty$ и $g_{-}^{\prime}(+\infty)=+\infty$.

Следующие предположения на функцию полезности $U$ являются классическими в финансовой математике: $U: \mathbf{R} \rightarrow \mathbf{R} \cup\{-\infty\}$ не убывает на $\mathbf{R}$, дифференцируема и строго вогнута на $\operatorname{int}\{x$ : 
$U(x)>-\infty\}=:(a,+\infty)$ и выполнены условия Инада: $\lim _{x \downarrow a} U^{\prime}(x)=+\infty$ и $\lim _{x \uparrow+\infty} U^{\prime}(x)=0$. Определим функцию

$$
V(y)=\sup _{x \in \mathbf{R}}[U(x)-x y], \quad y \in \mathbf{R} .
$$

Положим $W(x)=-U(-x), x \in \mathbf{R}$; если $a \in \mathbf{R}$, то переопределим при необходимости функцию $W$ в точке $-a$ так, чтобы она стала непрерывной слева в этой точке. Тогда $W \in \mathscr{W}$, и в силу неравенства $U(a) \leqslant-W(-a)$ имеет место соотношение

$$
V(y)=\sup _{x \in \mathbf{R}}[x y-W(x)], \quad y \in \mathbf{R},
$$

в частности, $V \in \mathscr{V}$.

Следствие 4. Функция полезности $U$ удовлетворяет перечисленным выше предположениям тогда и только тогда, когда $V \in \mathscr{V}$, int dom $V=(0,+\infty), V$ дифферениируема и строго выпукла на $(0,+\infty)$, $V_{+}^{\prime}(0)=+\infty$. При этом $a=-V_{-}^{\prime}(+\infty) u$

$$
U(x)=\inf _{y \in \mathbf{R}}[x y+V(y)], \quad x \neq a .
$$

До конца данного пункта будем предполагать, что фиксирована функция $f \in \mathscr{V}$, функция $g \in \mathscr{W}$ определена соотношением (11) (или (4)), а функция $F-$ соотношением (5). Положим $D=$ $\left\{(x, y) \in \mathbf{R}^{2}: y+g(x) \leqslant 0\right\}$.

Лемма 2. $D$ - непустое замкнутое выпуклое множество, $F-$ собственная полунепрерьвная снизу выпуклая функция, выполнено соотношение (6), и существует предел $\lim _{y \uparrow+\infty} f(y) / y$, совпадающий с $r_{g}$, a если $r_{f}=+\infty-$ также $c f_{-}^{\prime}(+\infty)$.

Д о к а з а т е л ь с т в о. По предложению $3 g$ - собственная полунепрерывная снизу выпуклая функция, поэтому ее надграфик

$$
\text { epi } g=\left\{(x, y) \in \mathbf{R}^{2}: x \in \operatorname{dom} g, y \geqslant g(x)\right\}
$$

есть непустое выпуклое замкнутое множество; то же верно для множества $D$, получаемого отражением множества ері $g$ относительно горизонтальной оси. По определению $F$ есть преобразование Фенхеля функции $\delta_{D}$, поэтому $F-$ полунепрерывная снизу выпуклая функция. Ясно, что $F(s, t)=+\infty$, если $t<0$. Пусть $t>0$, тогда

$$
F(s, t)=\sup _{x \in \operatorname{dom} g}[s x-t g(x)]=\sup _{x \in \mathbf{R}} t\left[\frac{s}{t} x-g(x)\right]=t f\left(\frac{s}{t}\right)
$$

в силу (12). Далее,

$$
F(s, 0)=\sup _{x \in \operatorname{dom} g} s x=\left\{\begin{array}{lll}
+\infty, & \text { если } & s<0 \\
0, & \text { если } & s=0 \\
s r_{g}, & \text { если } & s>0 .
\end{array}\right.
$$


В случае $r_{f}=+\infty$ по предложению 4 имеем $r_{g}=f_{-}^{\prime}(+\infty)$. Наконец, функция $F(1, t)$ выпукла и полунепрерывна снизу по $t$; по доказанному она равна $t f(1 / t)$ при $t>0$. Значит, в случае $r_{f}=+\infty$ она конечна при малых положительных $t$, следовательно, она непрерывна справа в нуле, откуда следует, что предел $\lim _{y \uparrow+\infty} f(y) / y$ существует и равен $r_{g}$. Если же $r_{f}<+\infty$, то предел, очевидно, существует и равен $+\infty$, в то время как $r_{g}=+\infty$ по предложению 4. Лемма 2 доказана.

5. Доказательство теоремы 1. Положим $\mathbf{D}=\left\{(\xi, \eta) \in \mathscr{L}^{\infty} \times\right.$ $\mathscr{L}^{\infty}:(\xi(\omega), \eta(\omega)) \in D$ для всех $\left.\omega\right\}$, где множество $D$ определено перед леммой 2. Для доказательства утверждения (i) достаточно заметить, что функция $\mathscr{J}_{f}(\mu, \nu)$ на $b a \times b a$ по определению есть преобразование Фенхеля функции $\delta_{\mathbf{D}}(\xi, \eta)$ на $\mathscr{L}^{\infty} \times \mathscr{L}^{\infty}$.

Утверждение (ii) есть непосредственное следствие определения $f$-дивергенции. Применяя (ii) к $\mathscr{G}=\{\varnothing, \Omega\}$, получаем левое неравенство в утверждении (vii). Далее, если $\eta(\omega)+g(\xi(\omega)) \leqslant 0$, то в силу определения функции $F$ и леммы 2 имеем $\xi(\omega) \leqslant F(1,0)=f(\infty) / \infty$, $\eta(\omega) \leqslant F(0,1)=f(0)$, откуда следует правое неравенство в утверждении (vii). Утверждения (viii) и (xi) следуют из (vii).

Пусть $\sigma$-алгебра $\mathscr{F}$ конечна и порождена разбиением $\Omega$ на дизъюнктные множества $A_{1}, \ldots, A_{n}$. Тогда

$$
\begin{aligned}
\mathscr{J}_{f}(\mu, \nu) & =\sup _{\substack{x_{1}, \ldots, x_{n}, y_{1}, \ldots, y_{n}: \\
y_{i}+g\left(x_{i}\right) \leqslant 0}} \sum_{i=1}^{n}\left[x_{i} \mu\left(A_{i}\right)+y_{i} \nu\left(A_{i}\right)\right] \\
& =\sum_{i=1}^{n} \sup _{x_{i}, y_{i}: y_{i}+g\left(x_{i}\right) \leqslant 0}\left[x_{i} \mu\left(A_{i}\right)+y_{i} \nu\left(A_{i}\right)\right]=\sum_{i=1}^{n} F\left(\mu\left(A_{i}\right), \nu\left(A_{i}\right)\right) .
\end{aligned}
$$

Возвращаясь к общему случаю, получаем из утверждения (ii), что левая часть (7) больше или равна правой. Для доказательства обратного неравенства достаточно рассмотреть случай $\mu, \nu \in b a_{+}$, иначе правая часть равна $+\infty$. Возьмем любое число $\alpha<\mathscr{J}_{f}(\mu, \nu)$, тогда по определению найдутся такие $\xi$ и $\eta$ из $\mathscr{L}^{\infty}$, что $\eta+g(\xi) \leqslant 0$ и $\beta:=\mu(\xi)+\nu(\eta)>\alpha$. Положим $\delta=(\beta-\alpha) /(\mu(\Omega)+\nu(\Omega))$ и возьмем такое конечное разбиение $\Omega$ на дизъюнктные множества $A_{1}, \ldots, A_{n} \in \mathscr{F}$, что $\left|\xi(\omega)-\xi\left(\omega^{\prime}\right)\right|<\delta$ и $\left|\eta(\omega)-\eta\left(\omega^{\prime}\right)\right|<\delta$, если $\omega$ и $\omega^{\prime}$ лежат в одном и том же элементе разбиения. Для каждого $i=1, \ldots, n$ возьмем точку $\omega_{i} \in A_{i}$ и положим $\tilde{\xi}(\omega)=\xi\left(\omega_{i}\right)$ и $\tilde{\eta}(\omega)=\eta\left(\omega_{i}\right)$, если $\omega \in A_{i}, i=1, \ldots, n$. Тогда $\tilde{\eta}+g(\tilde{\xi}) \leqslant 0$ и

$$
\begin{aligned}
\sum_{i=1}^{n} F\left(\mu\left(A_{i}\right), \nu\left(A_{i}\right)\right) & \geqslant \sum_{i=1}^{n}\left[\tilde{\xi}\left(\omega_{i}\right) \mu\left(A_{i}\right)+\tilde{\eta}\left(\omega_{i}\right) \nu\left(A_{i}\right)\right]=\mu(\tilde{\xi})+\nu(\tilde{\eta}) \\
& \geqslant \mu(\xi)+\eta(\nu)-\delta(\mu(\Omega)+\nu(\Omega))=\alpha .
\end{aligned}
$$

Утверждение (iii) доказано. 
Неравенство

$$
\mathscr{J}_{f}(\mu, \nu) \geqslant \sup _{\xi \in \mathscr{L}^{\infty}: g(\xi) \in \mathscr{L}^{\infty}}[\mu(\xi)-\nu(g(\xi))]
$$

очевидно, выполнено для любых $\mu, \nu \in b a$. Докажем обратное неравенство для $\mu, \nu \in b a_{+}$. Для этого заметим, что для $s, t \geqslant 0$

$$
F(s, t)=\sup _{x \in \operatorname{dom} g}[s x-\operatorname{tg}(x)] .
$$

Поэтому, как и в доказательстве утверждения (iii), для любого разбиения $\Omega$ на дизъюнктные множества $A_{1}, \ldots, A_{n} \in \mathscr{F}$

$$
\begin{aligned}
\sup _{\xi \in \mathscr{L}^{\infty}: g(\xi) \in \mathscr{L}^{\infty}}[\mu(\xi)-\nu(g(\xi))] & \geqslant \sup _{x_{1}, \ldots, x_{n} \in \operatorname{dom}} \sum_{i=1}^{n}\left[x_{i} \mu\left(A_{i}\right)-g\left(x_{i}\right) \nu\left(A_{i}\right)\right] \\
& =\sum_{i=1}^{n} F\left(\mu\left(A_{i}\right), \nu\left(A_{i}\right)\right) .
\end{aligned}
$$

С учетом утверждения (iii) получаем утверждение (vi).

Пусть функция $h$ определена как в утверждении (ix). Тогда сопряженная к ней функция $h^{*}$ связана с $g$ соотношением $h^{*}(x)=s g(x / t)$, и второе соотношение в утверждении (ix) следуют из определения $f$-дивергенции. Что касается первого соотношения, то оно очевидно.

В п. 2 приводилось доказательство утверждения (v), основанное на общих результатах Р. Рокафеллара. Здесь мы приведем прямое доказательство в духе доказательства теоремы 1.30 в [23]. Достаточно рассмотреть случай $\mu, \nu \in c a_{+}$, так как в противном случае обе части (9) равны $+\infty$. Учитывая положительную однородность функции $F$ (см. (6)), также достаточно предположить, что $\varrho=\mathrm{R}$ - вероятностная мера. Пусть $p=d \mu / d \mathrm{R}, q=d \nu / d \mathrm{R}$, а $\mathscr{G}$ - наименьшая $\sigma$-алгебра, относительно которой измеримы $p$ и $q$. Так как $\mathscr{G}$ счетно порождена, найдется такая возрастающая последовательность $\mathscr{G}_{1} \subseteq \cdots \subseteq \mathscr{G}_{n} \subseteq \cdots$ конечных $\sigma$-подалгебр, что $\mathscr{G}=\bigvee_{n} \mathscr{G}_{n}$. Сужения мер $\mathrm{R}, \mu, \nu$ на $\sigma$-алгебру $\mathscr{G}_{n}$ обозначим $\mathrm{R}_{n}, \mu_{n}, \nu_{n}$ соответственно, тогда $p_{n}:=d \mu_{n} / d \mathrm{R}_{n}=\mathbb{E}_{\mathrm{R}}\left(p \mid \mathscr{G}_{n}\right)$, $q_{n}:=d \nu_{n} / d \mathrm{R}_{n}=\mathbb{E}_{\mathrm{R}}\left(q \mid \mathscr{G}_{n}\right)$. По теореме Дуба о сходимости мартингалов при $n \rightarrow \infty$

$$
p_{n} \rightarrow \mathbb{E}_{\mathrm{R}}(p \mid \mathscr{G})=p, \quad q_{n} \rightarrow \mathbb{E}_{\mathrm{R}}(q \mid \mathscr{G})=q \quad \text { R-п.н. }
$$

В силу того, что $F$ полунепрерывна снизу и $F\left(p_{n}, q_{n}\right)$ ограничена снизу равномерно интегрируемой (относительно R) последовательностью $p_{n} x+$ $q_{n} y$ (где $(x, y)$ - произвольная фиксированная точка из $D$ ), из леммы Фату заключаем

$$
\begin{aligned}
\mathbb{E}_{\mathrm{R}} F(p, q) & \leqslant \mathbb{E}_{\mathrm{R}} \liminf _{n} F\left(p_{n}, q_{n}\right) \leqslant \liminf _{n} \mathbb{E}_{\mathrm{R}} F\left(p_{n}, q_{n}\right) \\
& =\liminf _{n} \mathscr{J}_{f}\left(\mu_{n}, \nu_{n}\right) \leqslant \mathscr{J}_{f}(\mu, \nu),
\end{aligned}
$$


где последнее неравенство вытекает из утверждения (ii). Остается заметить, что $F(p, q) \geqslant p \xi+q \eta$ для любой пары $(\xi, \eta) \in \mathbf{D}$, откуда $\mathbb{E}_{\mathrm{R}} F(p, q) \geqslant \mu(\xi)+\nu(\eta)$ для всех $(\xi, \eta) \in \mathbf{D}$.

Докажем утверждение (iv). Достаточно рассмотреть случай $\mu$, $\nu \in b a_{+}$. Неравенство $\mathscr{J}_{f}(\mu, \nu) \leqslant \mathscr{J}_{f}\left(\mu^{c}, \nu^{c}\right)+\mathscr{J}_{f}\left(\mu^{a}, \nu^{a}\right)$ следует из выпуклости и положительной однородности $f$-дивергенции (утверждения (i) и (ix)). Пусть вероятностная мера $\mathrm{P}$ доминирует счетноаддитивные меры $\mu^{c}$ и $\nu^{c}, p=d \mu^{c} / d \mathrm{P}, q=d \nu^{c} / d \mathrm{P}$. Так как меры $\mu^{a}$ и $\nu^{a}$ чисто конечно-аддитивны, по теореме 1.19 в [30] найдутся такие последовательности $\left\{B_{n}\right\}$ и $\left\{C_{n}\right\}$ множеств из $\mathscr{F}$, что $\mathrm{P}\left(B_{n}\right) \geqslant 1-1 / n$, $\mathrm{P}\left(C_{n}\right) \geqslant 1-1 / n, \mu^{a}\left(B_{n}\right)=0$ и $\nu^{a}\left(C_{n}\right)=0$ для любого $n$; переходя $\mathrm{k}$ конечным объединениям, можно считать эти последовательности возрастающими.

Возьмем произвольные числа $\alpha<\mathscr{J}_{f}\left(\mu^{c}, \nu^{c}\right), \delta>0$ и пару случайных величин $(\bar{\xi}, \bar{\eta}) \in \mathbf{D}$. Так как $\mathscr{J}_{f}\left(\mu^{c}, \nu^{c}\right)=\int_{\Omega} F(p, q) d \mathrm{P}$ по утверждению (v), при достаточно больших $n$

$$
\int_{B_{n} \cap C_{n}} F(p, q) d \mathrm{P}>\alpha \quad \text { и } \quad \int_{\Omega \backslash\left(B_{n} \cap C_{n}\right)}(\bar{\xi} p+\bar{\eta} q) d \mathrm{P}>-\delta .
$$

Пусть $n$ таково, что эти неравенства выполнены; положим $E=B_{n} \cap C_{n}$. Тогда

$$
\begin{aligned}
\mathscr{J}_{f}(\mu, \nu)=\sup _{(\xi, \eta) \in \mathbf{D}}\left[\mu^{c}(\xi)+\nu^{c}(\eta)+\mu^{a}(\xi)+\nu^{a}(\eta)\right] \\
=\sup _{(\xi, \eta) \in \mathbf{D}}\left[\mu^{c}\left(\xi \mathbf{1}_{E}\right)+\nu^{c}\left(\eta \mathbf{1}_{E}\right)+\mu^{c}\left(\xi \mathbf{1}_{\Omega \backslash E}\right)+\nu^{c}\left(\eta \mathbf{1}_{\Omega \backslash E}\right)\right. \\
\left.\quad \quad+\mu^{a}\left(\xi \mathbf{1}_{\Omega \backslash E}\right)+\nu^{a}\left(\eta \mathbf{1}_{\Omega \backslash E}\right)\right] \\
=\sup _{(\xi, \eta) \in \mathbf{D}}\left[\mu^{c}\left(\xi \mathbf{1}_{E}\right)+\nu^{c}\left(\eta \mathbf{1}_{E}\right)\right] \\
\quad+\left[\mu^{c}\left(\bar{\xi} \mathbf{1}_{\Omega \backslash E}\right)+\nu^{c}\left(\bar{\eta} \mathbf{1}_{\Omega \backslash E}\right)+\mu^{a}\left(\bar{\xi} \mathbf{1}_{\Omega \backslash E}\right)+\nu^{a}\left(\bar{\eta} \mathbf{1}_{\Omega \backslash E}\right)\right] .
\end{aligned}
$$

В силу утверждения (v)

$$
\sup _{(\xi, \eta) \in \mathbf{D}}\left[\mu^{c}\left(\xi \mathbf{1}_{E}\right)+\nu^{c}\left(\eta \mathbf{1}_{E}\right)\right]=\int_{E} F(p, q) d \mathrm{P}>\alpha
$$

поэтому

$$
\mathscr{J}_{f}(\mu, \nu) \geqslant \alpha-\delta+\mu^{a}(\bar{\xi})+\nu^{a}(\bar{\eta})
$$

В силу произвольности выбора $\alpha, \delta, \bar{\xi}, \bar{\eta}$ имеем

$$
\mathscr{J}_{f}(\mu, \nu) \geqslant \mathscr{J}_{f}\left(\mu^{c}, \nu^{c}\right)+\sup _{(\bar{\xi}, \bar{\eta}) \in \mathbf{D}}\left[\mu^{a}(\bar{\xi})+\nu^{a}(\bar{\eta})\right]=\mathscr{J}_{f}\left(\mu^{c}, \nu^{c}\right)+\mathscr{J}_{f}\left(\mu^{a}, \nu^{a}\right) .
$$

Заметим, что в предположениях утверждения (х) $f:=f_{1}+f_{2}-$ собственная полунепрерывная снизу выпуклая функция. В силу (6) $F$ есть 
сумма соответствующих функциям $f_{1}$ и $f_{2}$ функций $F_{1}$ и $F_{2}$, и утверждение $(\mathrm{x})$ легко следует из утверждений (iii) и (ii).

Пусть $\operatorname{dom} f \neq\{0\}$, т.е. функция $g$ не равна тождественно константе. Так как $g$ - выпуклая и полунепрерывная снизу функция, то ее надграфик ері $g$, как отмечалось в доказательстве леммы 2, есть выпуклое замкнутое множество. Рассмотрим отображение $(x, y) \rightsquigarrow$ $(-y,-x)$ плоскости на себя, т.е. отражение относительно биссектрисы второго и четвертого координатного углов. Удобно также представить это отображение как поворот плоскости по часовой стрелке на $90^{\circ}$ вокруг начала координат с последующим отражением относительно вертикальной оси. Легко видеть, что образ ері $g$ при этом отображении есть также надграфик некоторой собственной неубывающей функции, а именно функции

$$
\widehat{g}(x)=\inf \{y \in \mathbf{R}: g(-y) \leqslant-x\} \quad(\inf \varnothing=+\infty) .
$$

Так как отражение сохраняет свойства выпуклости и замкнутости, функция $\widehat{g}$ выпукла и полунепрерывна снизу, т.е. $\widehat{g} \in \mathscr{W}$. Из определения сразу получаем, что

$$
g(x)+y \leqslant 0 \Longleftrightarrow \widehat{g}(y)+x \leqslant 0, \quad(x, y) \in \mathbf{R}^{2} .
$$

Преобразование Фенхеля функции $\widehat{g}$ обозначим $\hat{f}$ и построим функцию $\widehat{F}$ с помощью соотношения (5), заменяя в нем $g$ на $\widehat{g}$. Из (13) ясно, что $F(x, y)=\widehat{F}(y, x)$, поэтому определенная нами функция $\hat{f}$ удовлетворяет $\hat{f}(y)=F(1, y), y \in \mathbf{R}$. Теперь утверждение (xii) очевидно. Теорема 1 доказана.

6. Заключительные замечания. Идею, лежащую в основе нашего определения $f$-дивергенции, можно использовать для определения более общего класса «выпуклых дивергенций», причем не только двух конечно-аддитивных мер, но и конечного их числа.

Именно, пусть задано произвольное множество $D \subseteq \mathbf{R}^{k}$. Обозначим $\left(\mathscr{L}^{\infty}\right)^{k}=\underbrace{\mathscr{L}^{\infty} \times \cdots \times \mathscr{L}^{\infty}}_{k \text { раз }}, b a^{k}=\underbrace{b a \times \cdots \times b a}_{k \text { раз }}$ и положим

$$
\mathbf{D}=\left\{\boldsymbol{\xi}=\left(\xi_{1}, \ldots, \xi_{k}\right) \in\left(\mathscr{L}^{\infty}\right)^{k}: \boldsymbol{\xi}(\omega) \in D_{\text {для всех } \omega\}}\right.
$$

Определим $D$-дивергенцию $\mathscr{J}_{D}\left(\mu_{1}, \ldots, \mu_{k}\right), \mu_{1}, \ldots, \mu_{k} \in b a$, как опорную функцию множества $\mathbf{D}$, т.е.

$$
\mathscr{J}_{D}\left(\mu_{1}, \ldots, \mu_{k}\right)=\sup _{\boldsymbol{\xi} \in \mathbf{D}}\left[\mu_{1}\left(\xi_{1}\right)+\cdots+\mu_{k}\left(\xi_{k}\right)\right]
$$

Определенная выше $f$-дивергенция является частным случаем $D$ дивергенции: возьмите $k=2$ и множество

$$
D=D(f)=\left\{(x, y) \in \mathbf{R}^{2}: y+g(x) \leqslant 0\right\}
$$


где $g$ - функция, сопряженная к $f$. Как отмечалось во введении, И. Чисар, Ф. Гамбоа и Э. Гассиа в статье [7] ввели понятие $\gamma$-дивергенции знакопеременной счетно-аддитивной меры $\mu$ и вероятностной меры $\nu$; эта $\gamma$-дивергенция есть не что иное, как $\mathscr{J}_{D}(\mu, \nu)$, где $D$ по-прежнему определяется соотношением (14), но от $f=\gamma$ требуется только выпуклость и полунепрерывность снизу, а эффективная область функции $f$ не обязательно является подмножеством положительной полупрямой. Отметим также, что понятие $D$-дивергенции охватывает понятие $f$-различия ( $f$-dissimilarity) $k$ вероятностных мер, введенное Л. Дьёрфи и Т. Неметцем [15], [16].

Следуя доказательству теоремы 1 , легко убедиться, что основные свойства $f$-дивергенций сохраняются для $D$-дивергенций. $\mathrm{B}$ частности: функция $\mathscr{J}_{D}\left(\mu_{1}, \ldots, \mu_{k}\right)$ на $b a^{k}$ принимает значения в $\mathbf{R} \cup\{+\infty\}$, выпукла и полунепрерывна снизу в топологии $\sigma\left(b a^{k},\left(\mathscr{L}^{\infty}\right)^{k}\right)$; $\mathscr{J}_{D}\left(\mu_{1}\left|\mathscr{G}, \ldots, \mu_{k}\right| \mathscr{G}\right) \leqslant \mathscr{J}_{D}\left(\mu_{1}, \ldots, \mu_{k}\right)$ для $\sigma$-подалгебры $\mathscr{G} \sigma$-алгебры $\mathscr{F} ;$

$$
\mathscr{J}_{D}\left(\mu_{1}, \ldots, \mu_{k}\right)=\sup \sum_{i=1}^{n} F_{D}\left(\mu_{1}\left(A_{i}\right), \ldots, \mu_{k}\left(A_{i}\right)\right), \quad \mu_{1}, \ldots, \mu_{k} \in b a,
$$

где $F_{D}$ есть опорная к множеству $D$ функция:

$$
F_{D}\left(t_{1}, \ldots, t_{k}\right)=\sup _{\left(x_{1}, \ldots, x_{k}\right) \in D}\left(t_{1} x_{1}+\cdots+t_{k} x_{k}\right), \quad\left(t_{1}, \ldots, t_{k}\right) \in \mathbf{R}^{k}
$$

а супремум берется по всем конечным разбиениям $\Omega$ на дизъюнктные множества $A_{1}, \ldots, A_{n} \in \mathscr{F}$;

$$
\mathscr{J}_{D}\left(\mu_{1}, \ldots, \mu_{k}\right)=\mathscr{J}_{D}\left(\mu_{1}^{c}, \ldots, \mu_{k}^{c}\right)+\mathscr{J}_{D}\left(\mu_{1}^{a}, \ldots, \mu_{k}^{a}\right), \quad \mu_{1}, \ldots, \mu_{k} \in b a
$$

И

$$
\mathscr{J}_{D}\left(\mu_{1}, \ldots, \mu_{k}\right)=\int_{\Omega} F_{D}\left(\frac{d \mu_{1}}{d \varrho}, \ldots, \frac{d \mu_{k}}{d \varrho}\right) d \varrho
$$

для любых счетно-аддитивных $\mu_{1}, \ldots, \mu_{k} \in c a$ и $\sigma$-конечной неотрицательной меры $\varrho$ на $(\Omega, \mathscr{F})$, доминирующей $\mu_{1}, \ldots, \mu_{k}$.

Следует отметить, что в определении $D$-дивергенции без ограничения общности можно считать множество $D$ замкнутым и выпуклым; более того, если нас интересуют только конечно-аддитивные меры с неотрицательными значениями, то можно еще считать, что $\mathbf{x} \in D$ влечет $\mathbf{y} \in D$ для всех $\mathbf{y} \leqslant \mathbf{x}$, где частичный порядок $\leqslant$ на $\mathbf{R}^{k}$ понимается покоординатно. Действительно, пусть $D \subseteq \mathbf{R}^{k}$ произвольно. Определим $D_{1}$ как выпуклое замыкание множества $D, D_{2}:=\bigcup_{\mathbf{x} \in D}\left\{\mathbf{y} \in \mathbf{R}^{k}: \mathbf{y} \leqslant \mathbf{x}\right\}$, $D_{3}$ - выпуклое замыкание $D_{2}$. Поскольку опорные функции множества и его выпуклого замыкания совпадают, имеем $F_{D}=F_{D_{1}}$ и $F_{D_{2}}=F_{D_{3}}$; легко также видеть, что $F_{D}$ и $F_{D_{2}}$ совпадают на $\mathbf{R}_{+}^{k}$. В силу (15) 
$\mathscr{J}_{D}$ и $\mathscr{J}_{D_{1}}$ совпадают на $b a^{k}$, а $\mathscr{J}_{D}$ и $\mathscr{J}_{D_{3}}$ - на $b a_{+}^{k}$ (при этом $\mathscr{J}_{D_{3}}\left(\mu_{1}, \ldots, \mu_{k}\right)=+\infty$, если $\mu_{i} \notin b a_{+}$для некоторого $\left.i\right)$.

В случае $k=2$ класс замкнутых выпуклых множеств $D$, для которых $\mathbf{x} \in D$ влечет $\mathbf{y} \in D$ для всех $\mathbf{y} \leqslant \mathbf{x}$, содержит все множества вида (14) с $g \in \mathscr{W}$, а помимо них - только плоскость $\mathbf{R}^{2}$ (что дает $D$ дивергенцию, тождественно равную $+\infty$ за исключением пары нулевых мер) и вертикальные полуплоскости $D=\{(x, y): x \leqslant a\}, a \in \mathbf{R}$ (в этом

случае $\mathscr{J}_{D}(\mu, \nu)=\mathscr{J}_{f}(\nu, \mu)$, где $f=a \delta_{\{0\}}$, ср. с утверждением (xii) теоремы 1). Таким образом, если ограничиться конечно-аддитивными мерами с неотрицательными значениями, для $k=2$ переход от $f$-дивергенций к $D$-дивергенциям не приводит к появлению новых содержательных объектов.

\section{СПИСОК ЛИТЕРАТУРЫ}

1. Алексеев B. М., Тихомиров В.М., Фомин С.В. Оптимальное управление. М.: Физматлит, 2005, 384 c.

2. Bednarski T. Binary experiments, minimax tests and 2-alternating capacities. - Ann. Statist., 1982, v. 10, № 1, p. 226-232.

3. Богачев В.И. Основы теории меры, т. 1. М.-Ижевск: НИЦ «Регулярная и хаотическая динамика», 2006, 583 с.

4. Broniatowski M., Keziou A. Minimization of $\phi$-divergences on sets of signed measures. - Studia Sci. Math. Hungar., 2006, v. 43, № 4, p. 403-442.

5. Csiszár I. Eine informationstheoretische Ungleichung und ihre Anwendung auf den Beweis der Ergodizität von Markoffschen Ketten. - Magyar Tud. Akad. Mat. Kutató Int. Közl., 1963, v. 8, p. 85-108.

6. Csiszár I. I-divergence geometry of probability distributions and minimization problems. - Ann. Probab., 1975, v. 3, № 1, p. 146-158.

7. Csiszár I., Gamboa F., Gassiat E. MEM pixel correlated solutions for generalized moment and interpolation problems. - IEEE Trans. Inform. Theory, 1999, v. 45, № 7 , p. 2253-2270.

8. Csiszár I., Tusnády G. Information geometry and alternating minimization procedures. - Statist. Decisions, 1984, suppl. 1, p. 205-237.

9. Cvitanić J., Schachermayer $W$., Wang $H$. Utility maximization in incomplete markets with random endowment. - Finance Stoch., 2001, v. 5, № 2, p. 259-272.

10. Delbaen F., Schachermayer $W$. A general version of the fundamental theorem of asset pricing. - Math. Ann., 1994, v. 300, № 3, p. 463-520.

11. Экланд И., Темам Р. Выпуклый анализ и вариационные проблемы. М.: Мир, $1979,399 \mathrm{c}$.

12. Föllmer H., Gundel A. Robust projections in the class of martingale measures. Illinois J. Math., 2006, v. 50, № 2, p. 439-472.

13. Goll T., Rüschendorf L. Minimax and minimal distance martingale measures and their relationship to portfolio optimization. - Finance Stoch., 2001, v. 5, № 4, p. 557-581.

14. Gundel $A$. Robust utility maximization for complete and incomplete market models. - Finance Stoch., 2005, v. 9, № 2, p. 151-176.

15. Györfi L., Nemetz T. f-dissimilarity: a general class of separation measures of several probability measures. - Topics in Information Theory (Second Colloq., Keszthely, 1975). Amsterdam: North-Holland, 1977, p. 309-321. (Colloq. Math. Soc. János Bolyai, v. 16.) 
16. Györfi L., Nemetz T. f-dissimilarity: a generalization of the affinity of several distributions. - Ann. Inst. Statist. Math., 1978, v. 30, № 1, p. 105-113.

17. Гущин $A . A . f$-дивергенция конечно-аддитивных мер. - Обозр. прикл. промышл. матем., 2005 , т. 12 , в. 3 , с. $657-658$.

18. Гущин А. А., Мордецки Э. Границы цен опционов для семимартингальных моделей рынка. - Тр. МИАН, 2002, т. 237, с. 80-122.

19. Huber P. J., Strassen V. Minimax tests and the Neyman-Pearson lemma for capacities. - Ann. Statist., 1973, v. 1, № 2, p. 251-263; correction: Ann. Statist., 1974, v. 2 , № 1 , p. 223-224.

20. Lembcke $J$. The necessity of strongly subadditive capacities for Neyman-Pearson minimax tests. - Monatsh. Math., 1988, v. 105, № 2, p. 113-126.

21. Léonard C. Minimizers of energy functionals. - Acta Math. Hungar., 2001, v. 93, № 4, p. 281-325.

22. Liese $F$. On the existence of $f$-projections. - Topics in Information Theory (Second Colloq., Keszthely, 1975). Amsterdam: North-Holland, 1977, p. 431-446. (Colloq. Math. Soc. János Bolyai, v. 16.)

23. Liese F., Vajda I. Convex Statistical Distances. Leipzig: Teubner, 1987, 224 p.

24. Невё Ж. Математические основы теории вероятностей. М.: Мир, 1969, 309 с.

25. Rockafellar R. T. Integrals which are convex functionals. - Pacific J. Math., 1968, v. 24 , № 3, p. 525-539.

26. Rockafellar R. T. Integrals which are convex functionals, II. - Pacific J. Math., 1971, v. 39, № 2, p. 439-469.

27. Рокафеллар Р. Выпуклый анализ. М.: Мир, 1973, 469 с.

28. Rüschendorf $L$. On the minimum discrimination information theorem. - Statist. Decisions, 1984, suppl. 1, p. 263-283.

29. Schied A., $W u$ C.-T. Duality theory for optimal investments under model uncertainty. - Statist. Decisions, 2005, v. 23, № 3, p. 199-217.

30. Yosida K., Hewitt E. Finitely additive measures. - Trans. Amer. Math. Soc., 1952, v. 72 , № 1 , p. $46-66$. 